ISSN 1112-9867

\title{
A LANGMUIR STUDY OF NOVEL SCHIFF BASE LIGAND FOR ION SENSOR APPLICATION
}

\author{
Y. Juahir ${ }^{2}$, B. M. Morris ${ }^{1}$, D. Lim ${ }^{1}$ and F. L. Supian ${ }^{1, *}$ \\ ${ }^{1}$ Department of Physics, Faculty of Science and Mathematics, Sultan Idris Education \\ University, 35900 Tanjong Malim, Perak, Malaysia \\ ${ }^{2}$ Department of Chemistry, Faculty of Science and Mathematics, Sultan Idris Education \\ University,35900 Tanjong Malim, Perak, Malaysia
}

Published online: 10 November 2017

\begin{abstract}
Two novel Schiff Base ligands are used in this work. These amphiphilic ligands were being chosen because of the suitability in forming Langmuir film by using Langmuir-Blodgett (LB) technique. Characterizations of these ligands were carried out through LB technique to obtain the surface pressure-mean molecular area (П-A) and surface potential-mean molecular area $(\Delta \mathrm{V}-\mathrm{A})$ isotherms. The analysis made from extrapolating the П-A graphs led to the result of the estimated area and the radius of the ligand molecules oriented on the air-water subphase. The UV-Visible spectrometer was used to study the optical properties of the ligands. This study was made in order to recognize the fundamental properties of these ligands for future works on detecting ions applications.
\end{abstract}

Keywords: Schiff Base Ligand; Langmuir-Blodgett technique; UV-Vis spectroscopy.

Author Correspondence, e-mail: faridah.lisa@fsmt.upsi.edu.my

doi: http://dx.doi.org/10.4314/jfas.v9i6s.10 


\section{INTRODUCTION}

Schiff bases are aldehyde - or ketone-like compounds in which the carbonyl group is replaced by an imine or azomethine group. It considers as a Schiff Base with the general structure, $\mathrm{R}_{2} \mathrm{C}=\mathrm{NR} \mathrm{R}^{\prime}\left(\mathrm{R}^{\prime} \neq \mathrm{H}\right)$. The nitrogen atom of azomethine $\mathrm{C}=\mathrm{N}$ double bond in Schiff base also exhibits a strong affinity for transition metal ions [1].

Schiff base is one of the famous compounds that is important in various field of science. The properties of Schiff base make them good ligands for metal ions detection. Schiff base metal complexes also have numerous applications like antitumor, antioxidant and possess attractive electronic properties. Several investigation have been done to investigatein situcoordination of Shiff base with metal ions at the air/water interface [2-3] and the complex proved to form a monolayer on the water surface. Schiff base also gave a good performance in molecular recognition [4] and formation of novel nanoarchitectures [2]. On the other hands, the Langmuir-Blodgett (LB) technique of monolayer transfer has often been used for the construction of highly ordered ultrathin film from water surfaces-to-solid substrates [5]. Hence, this technique has been chosen as the suitable technique to study the ligands in this research.

In this present work, novel Schiff base materials have been characterized using Langmuir and LB film. We also investigated the ultraviolet visible (UV-Vis) characteristics of both ligands in solution and thin film form.

\section{MATERIAL AND METHOD}

\subsection{Ligands}

In this work, two types of organic ligands had been used, 2-Hydroxy-5-bromobenzylidene-4-methylbenzenesulfonohydrazide (SBL-Br) in Fig. 1 and 2-Hydroxy-5-chlorobenzylidene-4-methylbenzenesulfonohydrazide (SBL-Cl). Both contain the amine group and made up of ketones or aldehyde. Both of the ligands have the same basic structure but difference in the end $(\mathrm{Br}$ or $\mathrm{Cl})$. 
<smiles>Cc1ccc(S(=O)(=O)N/N=C/c2cc(Br)ccc2O)cc1</smiles>

(a)<smiles>Cc1ccc(S(=O)(=O)N/N=C/c2cc(Cl)ccc2O)cc1</smiles>

(b)

Fig.1. Structure of (a) SBL-Br and (b) SBL-Cl

\subsection{Synthesis Process of Ligands}

Both SBL-Br and SBL-Cl was synthesis accordingly as inFig. 2.

\subsubsection{Process for the Synthesis of SBL-Br}

4-methylbenzenesulfonohydrazine $(0.30 \mathrm{~g}, 1.61 \mathrm{mmol})$ and 5-bromo-2-hydroxybenzaldehyde $(0.32 \mathrm{~g}, 1.61 \mathrm{mmol})$ were dissolved in acidified ethanol $(25 \mathrm{ml}, \mathrm{pH} 4.0-4.5)$ by adding of glacial acetic acid. The reactants were heated under reflux for 2 hours. Resulting colorless solution was evaporated in a vacuo to give brown crystalline precipitates which were then filtered off, washed with cold ethanol and then dried over silica gel (yield:72\%).

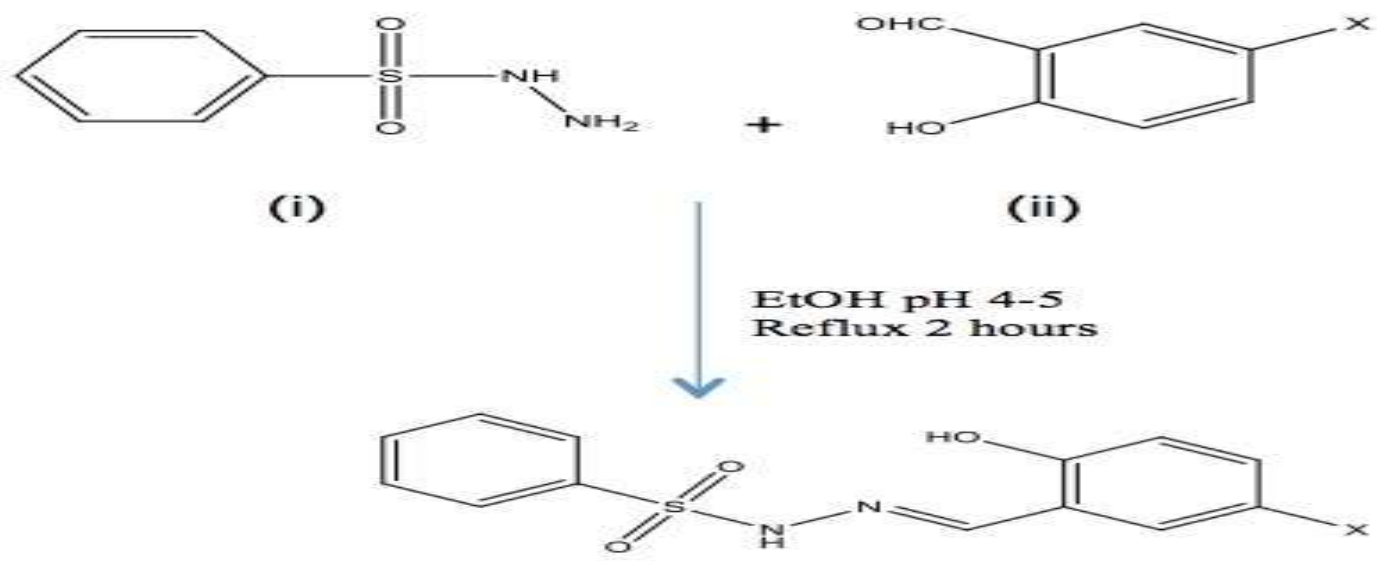

Fig.2. The synthesis of ligand SBL-Cl and SBL-Br, $(\mathrm{X}=\mathrm{Cl}$ or $\mathrm{Br})$ : (i)

4-Methylbenzenesulfonohydrazine; (ii) Salicylaldehyde

Process for the synthesis of SBL-Cl: SBL-Cl was prepared by refluxing a solution of 4-methylbenzenesulfonohydrazine $\quad\left(\begin{array}{lllllll}0.30 & \mathrm{~g}, & 0.30 & \mathrm{~g}, & 1.61 & \mathrm{mmol}\end{array}\right)$ and 5-chloro-2-hydroxybenzaldehyde $(0.25 \mathrm{~g}, 1.61 \mathrm{mmol})$ in acidified ethanol $(25 \mathrm{ml})$ for 2 hours. The resulting colorless solution was concentrated in a vacuo to give brown crystalline percipitates which were then filtered off, washed with cold ethanol and then dried over silica gel (Yield: 73\%). Then, the product obtained was recrystallized from 95\% ethanol. 


\subsection{Langmuir Blodgett (LB) studies}

Initially, beginning with SBL-Br, the ligands was mixed with $10 \mathrm{ml}$ of chloroform $\left(\mathrm{CHCl}_{3}\right)$ under 10 minutes of ultrasonification process $(40 \mathrm{kHz})$. Then, the solution of ligands (1.0 $\mathrm{mg} / \mathrm{ml}$ ) were carefully spread on the water surface using a micrometric syringe with several volumes (950 $\mu \mathrm{L}, 1000 \mu \mathrm{L}, 1050 \mu \mathrm{L}, 1100 \mu \mathrm{L}$ and $1150 \mu \mathrm{L})$ on the air- water interface of the LB trough. The method was repeated for SBL-Cl.

The water used for the monolayer experiments was deionized water $(18.2 \mathrm{M} \Omega / \mathrm{cm})$ that has been purified by Millipore Milli-Q system. A Wilhelmy plate made from filter paper act as a pressure sensor to measure the surface pressure (П) of the monolayer. After 15 minutes for evaporation of chloroform, the surface pressure-area (П-А) isotherms were recorded. The compression speeds of barriers were $5 \mathrm{~mm} / \mathrm{min}$ at the air/water subphase because interface lower speeds allow better molecular packing [6]. The П-A isotherm and surface potential of these materials, each had been analyzed using KSV 2000 system LB deposition trough and Surface Potential (S-POT) respectively. Surface pressure and surface potential studies have been carried out in a constant room temperature cleanroom because temperature has a direct effect on isotherm. The surface potential device (S-POT) connects directly to the existing LB surface. $\Delta$ Vsensor measures the potential difference above and below the film spontaneously, while the LB software measures the surface pressure. The alignment of the molecular dipoles could cause a large effect in the surface potential during compression as the orientation of the molecules change. Furthermore, the cleanroom ensure the experiment being carried out in a very clean place.

Meanwhile, UV-Vis measurements were carried out in $\mathrm{CHCl}_{3}$ solution using JASCO V-570 UV/VIS spectrophotometer with the path length of $1 \mathrm{~cm}$ and a spectral region from $190 \mathrm{~nm}$ to $1100 \mathrm{~nm}$.

\section{RESULTS AND DISCUSSION}

\subsection{Surface Pressure-Area (П-A) Isotherms of SBL-BrandSBL-Br}

The surface pressure against the mean molecular area isotherms of a floating layer indicates that the thermodynamic properties of molecules at the air/water interface. The monolayer at the air-water interface is manipulated by means of a movable barrier which had control the area per 
molecule. The result showed at Fig.3 demonstrated good stability and repeatability of the monolayers. As the solution spread on the subphase through differ in volume, the П-A curve shift towards left, revealing more ligands interact on air- water interphase. By extrapolating the steepest linear region, (acknowledging the solid phase) of the curve to zero surface pressure will gives the limiting area per molecule $\left(\mathrm{A}_{\mathrm{o}}\right)$ as shown in Table 1 and the radius is also being calculated.

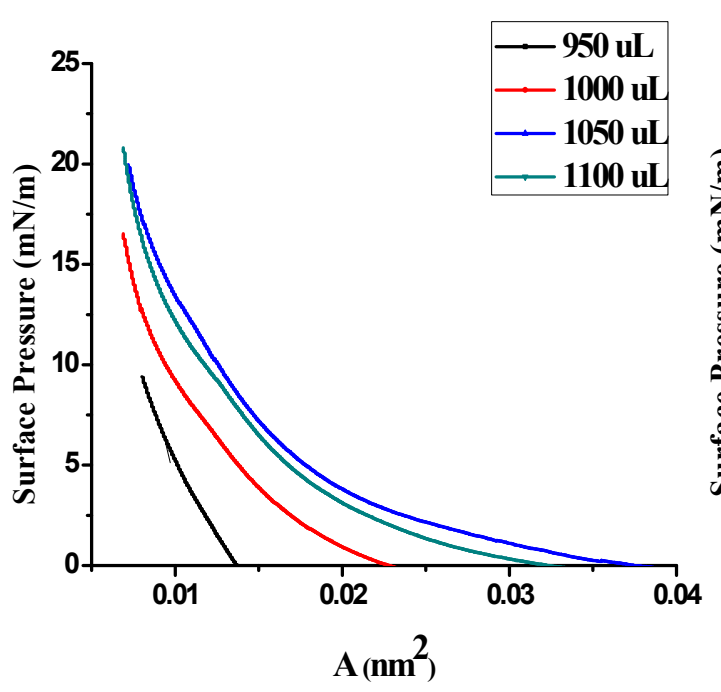

(a)

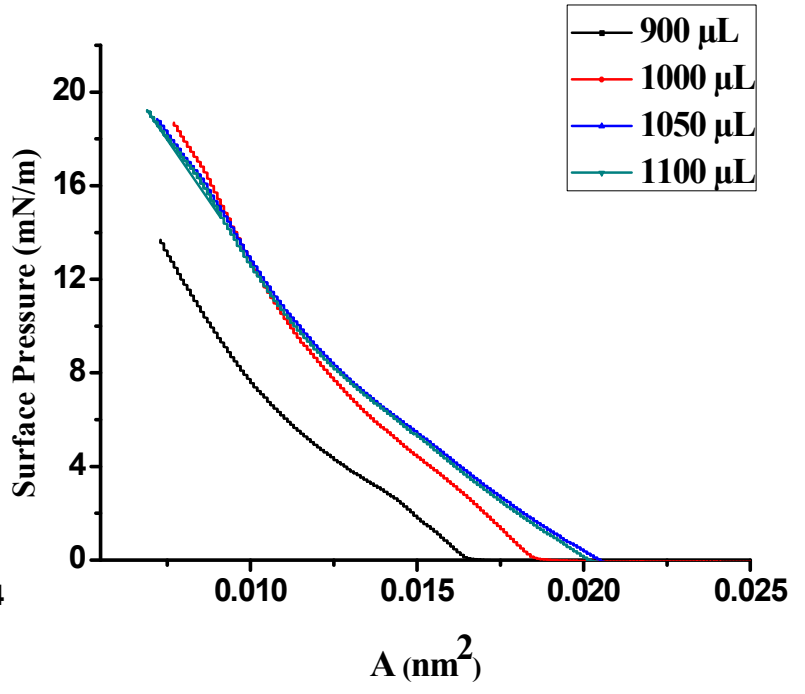

(b)

Fig.3. Surface pressure-area (П-A) isotherms of (a) SBL-Br and (b)SBL-Cl

Table 1. Mean molecular area and radius of SBL-Br and SBL-Cl

\begin{tabular}{ccccc}
\hline Volume & \multicolumn{2}{c}{ SBL-Br } & \multicolumn{2}{c}{ SBL-Cl } \\
$(\boldsymbol{\mu L})$ & Mean Molecule & Radius $(\mathbf{n m})$ & Mean Molecule & Radius \\
& Area, $\mathbf{A}_{\mathbf{0}}\left(\mathbf{n m}^{2}\right)$ & & Area, $\mathbf{A}_{\mathbf{0}}\left(\mathbf{n m}^{2}\right)$ & $(\mathbf{n m})$ \\
\hline 950 & $1.125 \times 10^{-2}$ & $6.0 \times 10^{-2}$ & $1.183 \times 10^{-2}$ & $6.1 \times 10^{-2}$ \\
1000 & $1.041 \times 10^{-2}$ & $5.8 \times 10^{-2}$ & $1.449 \times 10^{-2}$ & $6.8 \times 10^{-2}$ \\
1050 & $1.278 \times 10^{-2}$ & $6.4 \times 10^{-2}$ & $1.519 \times 10^{-2}$ & $7.0 \times 10^{-2}$ \\
1100 & $1.125 \times 10^{-2}$ & $6.0 \times 10^{-2}$ & $1.519 \times 10^{-2}$ & $7.0 \times 10^{-2}$ \\
\hline
\end{tabular}

The mean molecular area changed with different spreading volume on the subphase. The orientation of the molecule affects the surface pressure. Each of the molecular became packed, thus producing a semisolid monolayer due to compression from the barriers on the surface.

\subsection{Surface Potential and Effective Dipole Moment of Molecules at the Interface}


Fig. 4 depicts the $\Delta \mathrm{V}$-A versus area permolecule relationship for SBL- Br and SBL-Cl on a pure water subphase. The relationship can be seen at the onset of the surface potential where for the same volume dispersed $(1050 \mathrm{ul})$, maximum value occurs for SBL-Br at $0.018 \mathrm{~nm}^{2}$ for effective dipole moment and $0.021 \mathrm{~nm}^{2}$ for SBL-Cl. It can clearly be seen that SBL-Cl is higher compared to SBL-Br because of the presence of the conjugated electron system in SBL-Cl. This lead to a strong dipole moment value that is aligned orthogonally plane to the air-water interface. The relationship between $\Delta \mathrm{V}$ and effective dipole moment, $\mu_{\perp}$ is given by the Helmholtz equation in Equation(1).

$$
\Delta V \varepsilon_{0} \varepsilon A=\mu_{\perp}(1)
$$

where $\mathrm{A}$ is the area per molecule, $\varepsilon_{0}$ is the vacuum permittivity and $\varepsilon$ is the relative permittivity of the monolayer.

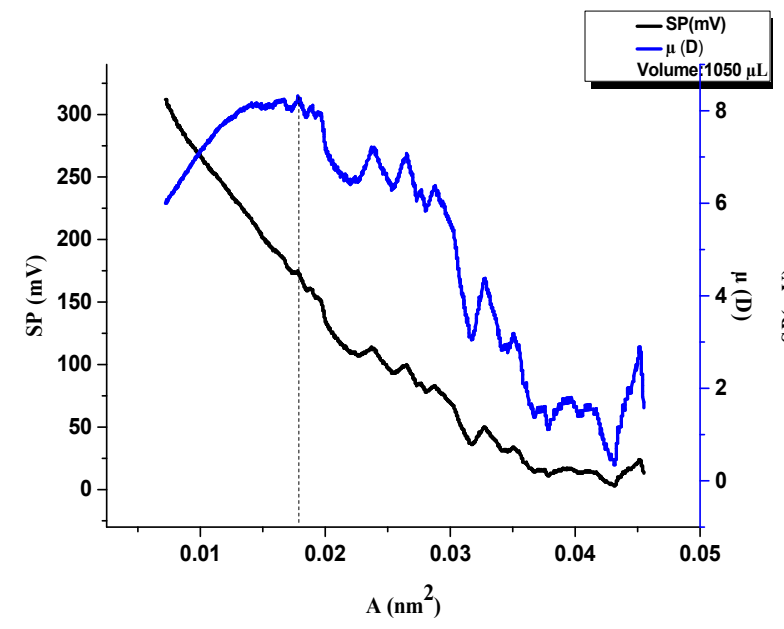

(a)

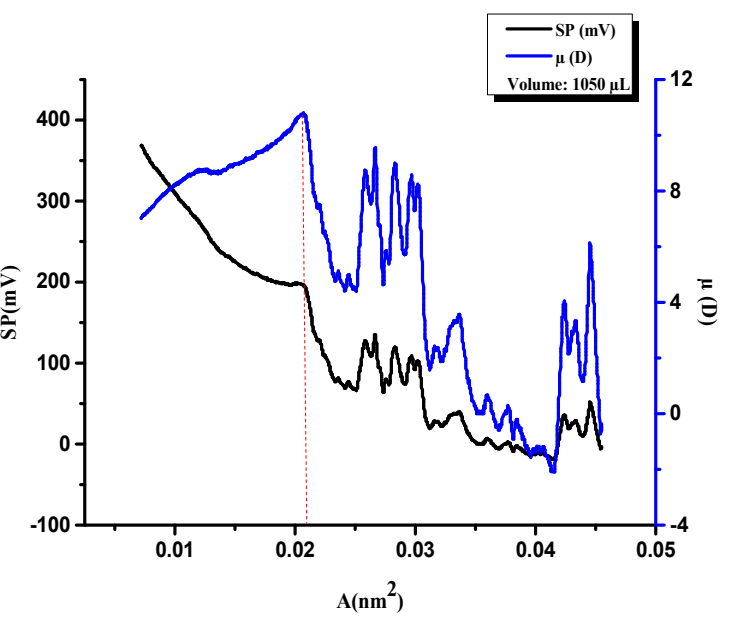

(b)

Fig.4.Surface potential and effective dipole moment of (a) SBL-Br and (b) SBL-Cl

\subsection{Ultraviolet-Visible Spectra Analysis}

The absorption spectra of SBL-Cl and SBL-Br in $\mathrm{CHCl}_{3}$ shows 3 main peaks (Fig. 5). The first and second peaks in SBL-Cl and SBL-Br which are $242 \mathrm{~nm}$ and $275 \mathrm{~nm}$ were due to the electronic transition of $\pi \rightarrow \pi^{*}$ of the aromatic rings or $\mathrm{C}=\mathrm{N}$ bond. While, the third peak of SBL- Cl $(332 \mathrm{~nm})$ and SBL-Br $(333 \mathrm{~nm})$ are due to the transition of $\mathrm{n} \rightarrow \pi^{*}$ of the imine group (-NH-N=C-) in the hydrazone ligand [7]. 


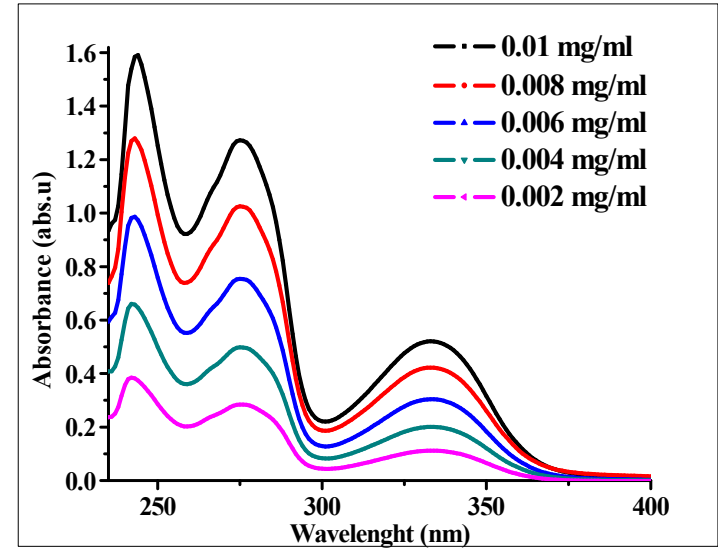

(a)

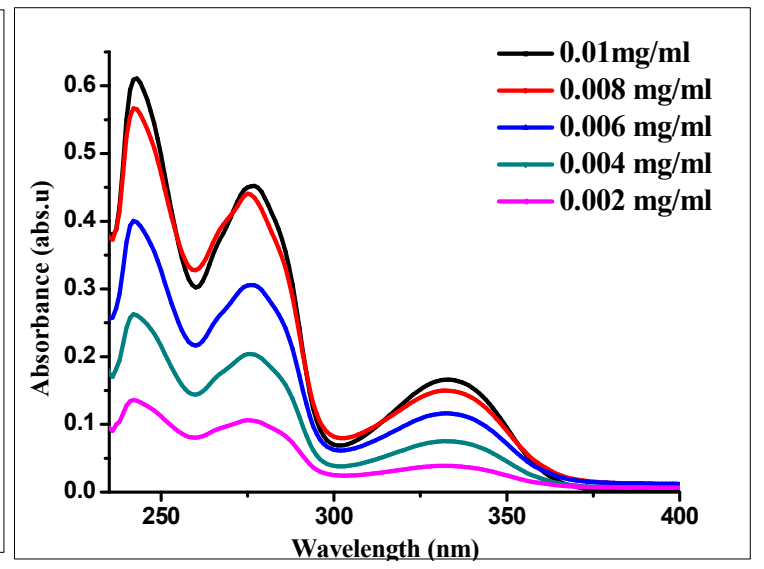

(b)

Fig.5. Absorption spectra of Ligand in chloroform, (a) SBL-Br and (b) SBL-Cl

By using Beer-Lambert Law, the molar absorptivity for both ligands were calculated in Fig.6using Equation(2).

$\varepsilon=\frac{A}{I C}(2)$

where $\varepsilon$ is the Greek letter, epsilon, A is the absorbance, 1 is the length of solution of the light passes through $(1 \mathrm{~cm})$ and $\mathrm{c}$ is the concentration of solution $\left(\mathrm{mol} \mathrm{dm} \mathrm{dm}^{-3}\right)$.

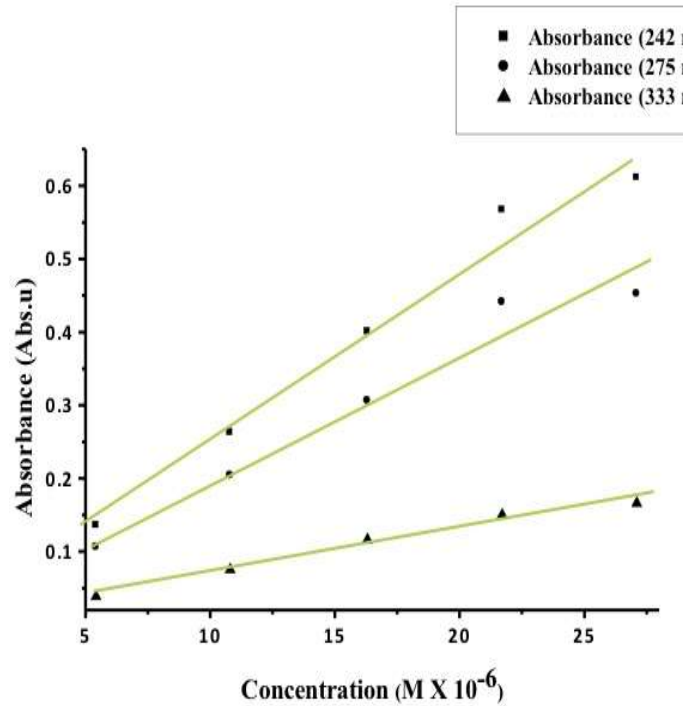

(a)

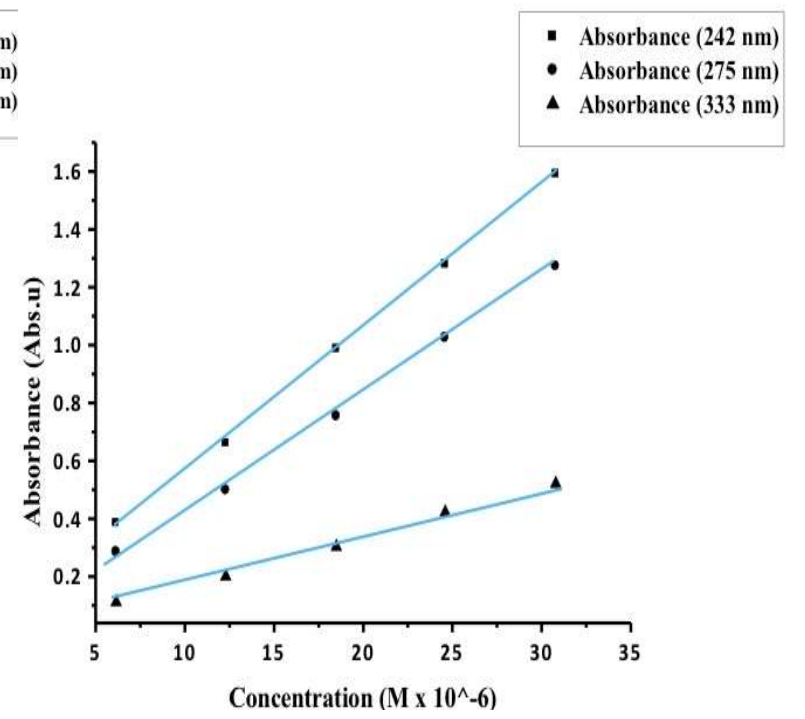

(b)

Fig.6. Absorption versus Concentration Graphs of (a) SBL-Br and (b) SBL-Cl

Table 2shows molar absorptivity $(\varepsilon)$ for the ligands for the same peak. It can be concluded that SBL-Cl has higher value, which means stronger absorbing chromophores compared to SBL-Br.

Table 2. Spectroscopic data for ligands in chloroform 


\begin{tabular}{|c|c|c|c|}
\hline \multicolumn{2}{|c|}{ SBL-Br } & \multicolumn{2}{c|}{ SBL-Cl } \\
\hline Absorption & Molar & Absorption & Molar \\
Maximum & Absorptivity, & Maximum & Absorptivity, \\
Wavelength & $\boldsymbol{\varepsilon}\left(\mathbf{M}^{-1} \mathbf{c m}^{-1}\right)$ & Wavelength & $\boldsymbol{\varepsilon}\left(\mathbf{M}^{-1} \mathbf{c m}^{-\mathbf{1}}\right)$ \\
\hline Peak 1 (242 nm) & 23110.8 & Peak 1 (242 nm) & 49227.4 \\
\hline Peak 2 (275 nm) & 17125.2 & Peak 2 (275 nm) & 40641.4 \\
\hline Peak 3 (332 nm) & 6072.7 & Peak 3 (333 nm) & 16881.9 \\
\hline
\end{tabular}

\section{CONCLUSION}

From the above data and discussion, the materials that investigated can form monolayers on the air-water interface. By comparison, SBL-Cl has higher values on the radius which were calculated using mean molecule area; higher value of effective dipole moment, $\mu_{\perp}$ and imply higher value of molar absorbtivity, $\boldsymbol{\varepsilon}$. It is suggested that this material of Schiff base ligand can be further study on thin film formation as a promising nanomaterials in sensing the heavy metals.

\section{ACKNOWLEDGEMENTS}

Financial support from RACE grant 2014-0003-102-62 and Sultan Idris Education University (UPSI) are acknowledged with gratitude.

\section{REFERENCES}

[1] Roy N, Pramanik HA, Paul PC, Singh TS. A highly sensitive and selective fluorescent chemosensor for detection of Zn 2+ based on a Schiff base. Spectrochimica Acta Part A: Molecular and Biomolecular Spectroscopy, 2015, 140:150-155

[2] Jiao T, Zhou J, Zhang L, Liu M. Supramolecular assembly and headgroup effect in interfacial organized films (I): A study of some bolaamphiphiles. Journal of Dispersion Science and Technology, 2011, 32(11):1592-1598 
[3] Jiao TF, Liu MH. Phase behaviors and 2D-3D morphological transition of aromatic Schiff base derivatives in organized molecular films. Acta Physico-Chimica Sinica, 2012, 28(6):1418-1424

[4] Bader NR. Applications of Schiff's bases chelates in quantitative analysis: A review. Rasayan Journal of Chemistry, 2010, 3(4):660-670

[5] Chen X, Xue QB, Yang KZ, Zhang QZ. Monolayers and Langmuir-Blodgett films of a liquid-crystalline polysiloxane with a Schiff base mesogenic unit in the side chain. Langmuir, 1995, 11(10):4082-4088

[6] Rubinger CP, Moreira RL, Cury LA, Fontes GN, Neves BR, Meneguzzi A, Ferreira CA. Langmuir-Blodgett and Langmuir-Schaefer films of poly (5-amino-1-naphthol) conjugated polymer. Applied Surface Science, 2006, 253(2):543-548

[7] Yusnita J, Puvaneswary S, Ali HM, Robinson WT, Kwai-Lin T. Synthesis, structural characterization and antibacterial activity of 2, 6-diacetylpyridine bis (benzenesulfonohydrazide) Schiff bases and their copper (II) complexes. Polyhedron, 2009, 28(14):3050-3054

\section{How to cite this article:}

Juahir Y, Morris B M, Lim D, Supian F L. A langmuir study of novel schiff base ligand for ion sensor application. J. Fundam. Appl. Sci., 2017, 9(6S), 115-123. 